\title{
DEFORMATIONS OF EXTENDED OBJECTS WITH EDGES
}

\author{
Riccardo Capovilla ${ }^{1}$ and Jemal Guven ${ }^{2 *}$ \\ ${ }^{1}$ Departamento de Física, Centro de Investigacion y de Estudios Avanzados del I.P.N. \\ Apdo Postal 14-740, 07000 México, D. F., MEXICO \\ 2 School of Theoretical Physics, D.I.A.S. \\ 10 Burlington Rd., Dublin 4, IRELAND
}

\begin{abstract}
We present a manifestly gauge covariant description of fluctuations of a relativistic extended object described by the Dirac-Nambu-Goto action with Dirac-Nambu-Goto loaded edges about a given classical solution. Whereas physical fluctuations of the bulk lie normal to its worldsheet, those on the edge possess an additional component directed into the bulk. These fluctuations couple in a non-trivial way involving the underlying geometrical structures associated with the worldsheet of the object and of its edge. We illustrate the formalism using as an example a string with massive point particles attached to its ends.
\end{abstract}

*Permanent address: Instituto de Ciencias Nucleares, Universidad Nacional Autónoma de México, Apdo. Postal 70-543, 04510, México, D.F., MEXICO 


\section{INTRODUCTION}

Relativistic extended objects with physical edges occur in many contexts. The simplest realization, often exploited to model hadrons consists of a string with particles attached to its ends (a zero dimensional edge). This model has obvious higher dimensional analogues: a membrane bounded by a string (a one dimensional edge) and so on. Once an edge is introduced it will generally possess its own degrees of freedom and capable of exchanging energy with the bulk. Indeed, to be democratic, these degrees of freedom should be treated on at least the same footing as those in the bulk. Often one might wish to focus on the edge degrees of freedom, integrating out the bulk degrees of freedom in favour of an action at a distance description of the edge dynamics. Such is the case in string models for QCD. What is clear is that the dynamics will generally depend sensitively on the specific interaction we admit between the bulk and edge degrees of freedom. The simplest action describing the dynamics of a relativistic extended object (membrane) with a non-null edge is a sum of two Dirac-Nambu-Goto [DNG] terms, i.e. one proportional to the area of its worldsheet, $m$, the other proportional to the area of the worldsheet, $\partial m$, of its boundary. The interaction induced between the bulk and the edge by this action is local. While it is perhaps over optimistic to expect this model to describe realistic physical systems, it does provide a useful point of departure for more sophisticated study.

In earlier work, we demonstrated that the interaction between the dynamical degrees of freedom on the edge and those in the bulk can be cast in a universal geometrical form [1,22]. Carter has also addressed the problem from a different point of view recognising that both the bulk equations of motion and the boundary equations could be cast in a single generalized 'sail' equation [3]. Earlier relevant work in the context of string models for QCD is summarized in Ref. [4]. For the case of hybrid topological defects in cosmology, see Ref. [5]. The dynamics in the interior is described by the system of non-linear hyperbolic partial differential equations:

$$
K^{i}=0
$$

Here $K^{i}$ is the trace of the $i^{\text {th }}$ extrinsic curvature of $m$ embedded in spacetime, one for each co-dimension of the embedding. These equations are unchanged with respect to those which correspond to extended objects with empty boundary. The interior worldsheet is always extremal. It is the coupling at the boundary which is non-trivial. Each edge must satisfy

$$
\mu_{b} k=-\mu,
$$

where $k$ is the trace of the extrinsic curvature of the boundary worldsheet $\partial m$ embedded as a hypersurface in $m$. The tension in the membrane is $\mu$, that in its boundary is $\mu_{b}$ (if pointlike, this will be a mass). Eq.(2) is the only place where the tensions feature explicitly. The boundary worldsheet has constant mean extrinsic curvature.

The edge in turn informs the bulk how to move by constraining the projected extrinsic curvatures of $m$ to vanish on $\partial m$,

$$
\mathcal{H}^{a b} K_{a b}^{i}=0
$$

where $\mathcal{H}^{a b}$ denotes the projector from $m$ onto $\partial m$. In [2], we emphasized the different roles played by Eq.(2) and Eqs. (3) in mediating the interaction between the bulk and the edge degrees of freedom. 
The system of equations (11), (2) and (3) is simultaneously worldsheet/boundary worldsheet diffeomorphism covariant. Unfortunately, however, the system is untractable unless a large degree of symmetry is imposed. Even for a string bounded by point particles, the coupling to the particles spoils the linearity of the problem.

In this paper, we propose to examine the interaction between the bulk and the edge perturbatively: at face value, this simply involves linearizing the equations of motion, (11) to (3), about a given classical solution. In practice, however, it is not so straightforward. The challenge here is to cast the linearized equations, like the equations of motion, in a manifestly covariant geometrical form.

We already know how to describe fluctuations in the bulk for a DNG extended object [6 9]. We know that physical fluctuations there correspond to normal deformations of the bulk worldsheet. There is therefore one for each co-dimension associated with the embedding of the worldsheet in spacetime. These quantities satisfy a system of coupled hyperbolic partial differential equations with support on the worldsheet of the undeformed object. The principal subtlety in the description adopted in Ref. [6] lies in the identification of the role of the twist potential of the background worldsheet in ensuring covariance under worldsheet normal rotations.

On the edge there is an additional possible mode of fluctuation directed into (or out of) the bulk, tangent to the bulk worldsheet. These edge fluctuations will couple to fluctuations in the bulk. They satisfy a hyperbolic partial (ordinary if pointlike) differential equation with support on the worldsheet of the undeformed edge and with a source linear in the boundary values of the bulk modes. The boundary conditions on the bulk fluctuations are themselves dynamical, assuming the form of a system of coupled hyperbolic partial differential equations with support on the worldsheet of the undeformed edge with source linear in the edge mode. Clearly, this is an intricately coupled system. Indeed, generically, it is not possible to decouple them. Neither pure edge modes nor pure bulk modes with an independent dynamical life are supported by the system.

To derive the linearized equations of motion, our strategy is to first control technicalities of a kinematical nature. For this reason, sect.II is devoted to a derivation of some kinematical identities in the manner undertaken in Ref. [9] for the parent worldsheet. Specifically, we examine the deformations in the edge geometry, both intrinsic and extrinsic, which are induced by a deformation in the edge worldsheet. There are two ways this deformation can be approached. The approach adopted here exploits the possibility of describing the edge worldsheet itself as an embedding in spacetime by forming the composition of embeddings: embedding first edge worldsheet in the bulk worldsheet followed by the embedding of the bulk worldsheet in spacetime. The alternative approach (the one adopted in Ref. [2] for calculations) focuses on the boundary behavior of the bulk worldsheet. While this straightforward approach was adequate in that context, where we needed only consider deformations of intrinsic geometry, its shortcomings become apparent as soon as one moves on to treat deformations of the extrinsic geometry. Indeed the approach adopted here not only facilitates calculations, but it also further elucidates the geometrical nature of the coupling between edge and bulk fluctuations.

We end with an application of our formalism. We first specialize to the case of a string bounded by massive point particles. We then examine the special case of the motion of a rigidly rotating string with particles attached to its ends. We show that, contrary to 
naive Newtonian expectations, which would suggest the existence of a breathing mode corresponding to the motion of a particle in a linear potential, the only such motion is circular corresponding to a timelike right circular helicoid. We note that the string equation of motion is not among the set of equations of motion corresponding to a truncation of the action to the symmetry appropriate to rigid motion. We examine how this system behaves under perturbations. We find that the normal modes of the perturbations have complex frequencies.

For the sake of simplicity, in the text we consider only a flat background spacetime. We relegate the equations corresponding to the case of an arbitrary background spacetime to an Appendix.

\section{EMBEDDING OF THE EDGE WORLDSHEET IN SPACETIME}

In Ref. [2], we showed that, with respect to an adapted basis of normal vectors, both the equations of motion (2) and the boundary conditions (3) can be cast entirely in terms of the extrinsic geometry associated with the direct embedding of the edge worldsheet in spacetime. This was treated as a curiosity in Ref. [2]. Because of the central role this shift in perspective will play in the sequel, it is worthwhile to recall the treatment in Ref. [2], elaborating when appropriate.

The embedding of the bulk worldsheet, $m$, in spacetime, induces an embedding in spacetime of the edge worldsheet as follows: we identify the edge worldsheet with the timelike boundary of $m$ ( $\partial m$ say). This is described by the embedding $\xi^{a}=\chi^{a}\left(u^{A}\right)$ $(a=0,1, \cdots, D-1, A=0,1, \cdots, D-2)$ in $m . m$ in turn is described by the embedding in spacetime, $x^{\mu}=X^{\mu}\left(\xi^{a}\right),(\mu=0,1, \cdots, N-1)$. The composition of these two embeddings yields an embedding of the edge worldsheet in spacetime,

$$
x^{\mu}=\bar{X}^{\mu}\left(u^{A}\right),
$$

where

$$
\bar{X}^{\mu}\left(u^{A}\right)=X^{\mu}\left(\xi^{a}\left(u^{A}\right)\right)
$$

We denote the tangent vectors to $m$ in spacetime, $e_{a}^{\mu}$; the tangent vectors to the edge worldsheet in $m$ associated with $\chi, \epsilon_{A}^{a}$. The latter can be promoted to spacetime vectors associated with the embedding $\bar{X}$,

$$
f_{A}^{\mu}:=e^{\mu}{ }_{a} \epsilon_{A}^{a} .
$$

The metric $h_{A B}$ induced directly from spacetime by the embedding $\bar{X}^{\mu}$ coincides with that induced from $m$ by the embedding $\chi^{a}$ :

$$
h_{A B}=g_{\mu \nu} f_{A}^{\mu} f_{B}^{\nu}=\gamma_{a b} \epsilon_{A}^{a} \epsilon_{B}^{b} .
$$

To construct the extrinsic geometry associated with the embedding $\bar{X}^{\mu}$, we need to erect the normals to $\partial m$ in spacetime. The choice we make exploits the fact that the spacetime normals to $m,\left\{n^{\mu i}\right\}$ are also normal to $\partial m$ in spacetime $(i, j, \cdots=1, \cdots, N-D)$. We take $\left\{n^{\mu i}\right\}$ to satisfy $g_{\mu \nu} n^{\mu i} n^{\nu j}=\delta^{i j}$. We now promote the unit normal to $\partial m$ into $m$ 
to a spacetime vector with $\eta^{\mu}:=f^{\mu}{ }_{a} \eta^{a}$. The vector $\eta^{\mu}$ is clearly normal to the $\left\{n^{\mu i}\right\}$ in spacetime. We how have a complete orthonormal basis of normal vectors, which we label $m^{I}:=\left\{m^{0}, m^{i}\right\}=\left\{\eta, n^{i}\right\}$, such that $g_{\mu \nu} m^{\mu I} m^{\nu J}=\delta^{I J}(I, J, \cdots=1, \cdots, N-D+1)$. We use the index 0 to denote the direction along $\eta^{\mu}$. It should not be confused with a timelike index. In adapting our choice of normals in this way, we surrender the full $O(N-D+1)$ gauge freedom associated with rotations of an arbitrarily chosen set of normal vectors, $\left\{m^{\mu I}\right\}$, for the $O(N-D)$ rotation freedom possessed by the $\left\{n^{\mu i}\right\}$.

We now write down the Gauss-Weingarten equations associated with the embedding (5):

$$
\begin{aligned}
D_{A} f_{B} & =\gamma_{A B}{ }^{C} f_{C}-L_{A B}{ }^{I} m_{I}, \\
D_{A} m^{I} & =L_{A B}{ }^{I} f^{B}+\sigma_{A}{ }^{I J} m_{J} .
\end{aligned}
$$

Here $D_{A}:=f^{\mu}{ }_{A} D_{\mu}$, with $D_{\mu}$ the spacetime covariant derivative. The connection $\gamma_{A B}{ }^{C}$ is the one compatible with $h_{A B}$. The quantity $L_{A B}{ }^{I}=L_{B A}{ }^{I}$ is the $I^{\text {th }}$ extrinsic curvature of $\partial m$ embedded in spacetime, and $\sigma_{A}{ }^{I J}$ its extrinsic twist potential.

With respect to the adapted basis, it is simple to check that

$$
L_{A B}^{i}=\epsilon_{A}^{a} \epsilon_{B}^{b} K_{a b}^{i}=K_{A B}{ }^{i},
$$

and that

$$
\sigma_{A}^{i j}=\epsilon_{A}^{a} \omega_{a}^{i j}=\omega_{A}^{i j} .
$$

The boundary inherits the extrinsic curvature $K_{a b}{ }^{i}$ and extrinsic twist $\omega_{a}^{i j}$ associated with the embedding of the parent worldsheet $m$ in spacetime.

The component of the extrinsic curvature directed along the normal $\eta^{\mu}$ is the extrinsic curvature associated with the embedding of the edge in the bulk:

$$
L_{A B}{ }^{0}=k_{A B} .
$$

Thus

$$
k=h^{A B} k_{A B}=h^{A B} L_{A B}^{0} .
$$

Recalling that $\mathcal{H}^{a b}=h^{A B} \epsilon^{a}{ }_{A} \epsilon^{b}{ }_{B}$ is the projector from the bulk onto the edge, we also have

$$
\mathcal{H}^{a b} K_{a b}{ }^{i}=h^{A B} L_{A B}{ }^{i} .
$$

It is now clear that Eqs.(2) and (3) can be recast as

$$
\mu_{b} L^{I}=-\mu \delta^{I 0} .
$$

To complete the description of the boundary twist, we require $\sigma_{A}{ }^{i 0}$. This is constructed from the tangent-normal projection of the bulk worldsheet extrinsic curvature as

$$
\sigma_{A}^{i 0}=\eta^{a} \epsilon_{A}^{b} K_{a b}^{i}=: K_{A}^{i} .
$$

Thus, part of the bulk worldsheet extrinsic curvature gets cast up on the boundary as a component of the twist potential. Henceforth we will denote this projection with $K_{A}{ }^{i}$. The fact that $\sigma_{A}{ }^{i 0}$ is completely determined is consistent with the fact that we have surrendered the freedom to rotate $\eta^{\mu}$ into the $n_{i}^{\mu}$ s. Adapting the basis amounts to a partial gauge choice. We note that the only projections of the bulk worldsheet extrinsic curvature which have not been picked up by the boundary extrinsic geometry are the $K_{a b}^{i} \eta^{a} \eta^{b}$. For a DNG-DNG system this projection vanishes on the boundary and therefore the entire parent worldsheet extrinsic geometry in the neighborhood of the boundary is encoded completely in the boundary twist. 


\section{DEFORMING THE EDGE GEOMETRY}

Let us consider a deformation of the edge worldsheet, $\partial m$, described by the infinitesimal deformation in the embedding (4),

$$
\bar{X}^{\mu} \rightarrow \bar{X}^{\mu}+\delta \bar{X}^{\mu}
$$

We can decompose it with respect to the spacetime basis adapted to the edge,

$$
\delta \bar{X}=\phi_{I} m^{I}+\phi^{A} f_{A}
$$

i.e. in parts normal and tangential to $\partial m$, respectively. The latter term corresponds to a boundary worldsheet diffeomorphism which we subsequently drop, since we will be interested only in deformations of quantities invariant under reparameterizations of the edge worldsheet. The normal deformation decomposes naturally into two parts: one part normal to the parent worldsheet, the other directed into it. We write:

$$
\phi^{I} m_{I}=\psi \eta+\phi^{i} n_{i} .
$$

$\phi^{i}$ can be identified with the value assumed on the boundary of the projection along the worldsheet normal $n_{i}$ of a deformation occuring in the bulk:

$$
\begin{aligned}
X^{\mu} & \rightarrow X^{\mu}+\delta X^{\mu}, \\
\delta X & =\Phi^{i} n_{i}+\Phi^{a} e_{a}, \\
\phi^{a} & =\left.\Phi^{a}\right|_{\partial m}=\phi^{A} \epsilon_{A}^{a}+\psi \eta^{a}, \\
\phi^{i} & =\left.\Phi^{i}\right|_{\partial m} .
\end{aligned}
$$

We will subsequently refer to $\Phi^{i}$ as the bulk normal deformation. ¿From the point of view of the parent worldsheet, $\psi$ corresponds to a piece of its tangential deformation; we will subsequently refer to it as the edge normal deformation. Note that we are not allowed to drop the tangential part of the deformation of the bulk worldsheet, since the presence of a non-empty boundary breaks diffeomorphism invariance. This is perhaps best appreciated if one interprets diffeomorphisms as active transformations which can push a point off the edges.

We can now exploit the general formalism developed in [9] to describe deformations of an arbitrary worldsheet, and apply it to the deformation of the edge geometry.

To simplify our treatment, in the text we will consider only the case of a flat background spacetime. The modified expressions one obtains in the case of an arbitrary background spacetime are given in an Appendix.

We have that, under the deformation (19) the metric varies according to

$$
D_{\delta \bar{X}_{\perp}} h_{A B}=2 L_{A B}^{I} \phi_{I}=2 K_{A B}^{i} \phi_{i}+2 k_{A B} \psi
$$

where we use the deformation operator

$$
D_{\delta \bar{X}_{\perp}}:=\left(\delta \bar{X}^{\mu}\right)_{\perp} D_{\mu}=\phi^{I} m^{\mu}{ }_{I} D_{\mu}=\psi \eta^{\mu} D_{\mu}+\phi^{i} n^{\mu}{ }_{i} D_{\mu} .
$$

The extrinsic curvature of the edge as embedded in spacetime transforms as follows [9]: 


$$
\hat{D}_{\delta \bar{X}_{\perp}} L_{A B}^{I}=-\hat{\mathcal{D}}_{A} \hat{\mathcal{D}}_{B} \phi^{I}+L_{A C}{ }^{I} L^{C}{ }_{B J} \phi^{J} .
$$

On the right hand side we use the covariant derivative associated with the connection $\sigma_{A}{ }^{I J}$,

$$
\hat{\mathcal{D}}_{A} \phi^{I}=\mathcal{D}_{A} \phi^{I}-\sigma_{A}^{I J} \phi_{J}
$$

where $\mathcal{D}_{A}$ is the covariant derivative on the edge, compatible with $h_{A B}$. The hat appearing over the deformation operator $D_{\bar{X}_{\perp}}$ is the analogue for normal deformations of $\bar{X}^{\mu}$ of the totally covariant operator $\tilde{\mathcal{D}}_{\delta}$ for normal deformations of $X^{\mu}$ which was introduced in Ref. [9]. Its significance will be explained below.

Our strategy is to first decompose Eq.(23) into mutually orthogonal parts with respect to the decomposition (19). We express the $I=0$ component of Eq.(23) as

$$
\hat{D}_{\delta \bar{X}_{\perp}} L_{A B}{ }^{0}=\hat{D}_{\delta \bar{X}_{\perp}} k_{A B}=-\hat{\mathcal{D}}_{A} \hat{\mathcal{D}}_{B} \psi+k_{A C} k_{B}^{C} \psi+k_{A C} K_{B i}^{C} \phi^{i} .
$$

We now exploit the fact that

$$
\hat{\mathcal{D}}_{A} \phi^{i}=\widetilde{\mathcal{D}}_{A} \phi^{i}-K_{A}{ }^{i} \psi
$$

where we have used the notation

$$
\widetilde{\mathcal{D}}_{A} \phi^{i}:=\epsilon_{A}^{a} \widetilde{\nabla}_{a} \phi^{i}=\epsilon_{A}^{a}\left[\nabla_{a} \phi^{i}-\omega_{a}^{i j} \phi_{j}\right],
$$

and that

$$
\hat{\mathcal{D}}_{A} \psi=\mathcal{D}_{A} \psi+K_{A}^{i} \phi_{i} .
$$

With the help of these expressions, we can decompose the first term appearing in Eq.(25),

$$
\begin{aligned}
\hat{\mathcal{D}}_{A} \hat{\mathcal{D}}_{B} \psi & =\mathcal{D}_{A}\left(\mathcal{D}_{B} \psi+K_{B}^{i} \phi_{i}\right)+K_{A}^{i}\left(\widetilde{\mathcal{D}}_{B} \phi_{i}-K_{i B} \psi\right) \\
& =\mathcal{D}_{A} \mathcal{D}_{B} \psi+2 K_{(A}^{i} \widetilde{\mathcal{D}}_{B)} \phi_{i}+\left(\widetilde{\mathcal{D}}_{A} K_{B}^{i}\right) \phi_{i}-K_{A}^{i} K_{i B} \psi,
\end{aligned}
$$

so that Eq. (25) can now be written in the form

$$
\begin{aligned}
\hat{D}_{\delta \bar{X}_{\perp}} k_{A B}= & -\mathcal{D}_{A} \mathcal{D}_{B} \psi+\left[K_{A}^{i} K_{i B}+k_{A C} k_{B}^{C}\right] \psi \\
& -2 K^{i}{ }_{(A} \widetilde{\mathcal{D}}_{B)} \phi_{i}-\left(\widetilde{\mathcal{D}}_{A} K_{B}^{i}\right) \phi_{i}+k_{A C} K_{B i}^{C} \phi^{i} .
\end{aligned}
$$

We can exploit Eq.(21), together with Eq.(30) to obtain for the deformation of the trace,

$$
\begin{aligned}
\hat{D}_{\delta \bar{X}_{\perp}} k= & -\mathcal{D}_{A} \mathcal{D}^{A} \psi+\left[K_{A i} K^{A i}-k_{A B} k^{A B}\right] \psi \\
& -2 K_{A}{ }^{i}\left(\widetilde{\mathcal{D}}^{A} \phi_{i}\right)-\left(\widetilde{\mathcal{D}}_{A} K^{A i}\right) \phi_{i}-k_{A B} K^{A B}{ }_{i} \phi^{i} .
\end{aligned}
$$

Let us now consider the transformation of the remaining projections, $L_{A B}{ }^{i}$. ¿From Eq. (23), we have that

$$
\hat{D}_{\bar{X}_{\perp}} L_{A B}{ }^{i}=\hat{D}_{\bar{X}_{\perp}} K_{A B}{ }^{i}=-\hat{\mathcal{D}}_{A} \hat{\mathcal{D}}_{B} \phi^{i}+K_{A C}{ }^{i} k^{C}{ }_{B} \psi+K_{A C}{ }^{i} K^{C}{ }_{B j} \phi^{j} .
$$

Here, using Eqs. (26), (28), we can express the first term on the right hand side as 


$$
\begin{aligned}
\hat{\mathcal{D}}_{A} \hat{\mathcal{D}}_{B} \phi^{i} & =\widetilde{\mathcal{D}}_{A}\left(\widetilde{\mathcal{D}}_{B} \phi^{i}-K_{B}{ }^{i} \psi\right)-K_{A}{ }^{i}\left(\mathcal{D}_{B} \psi-K_{B}{ }^{j} \phi_{j}\right) \\
& =\widetilde{\mathcal{D}}_{A} \widetilde{\mathcal{D}}_{B} \phi^{i}-2 K_{(A}{ }^{i} \mathcal{D}_{B)} \psi-\left(\widetilde{\mathcal{D}}_{A} K_{B}{ }^{i}\right) \psi-K_{A}{ }^{i} K_{B j} \phi^{j}
\end{aligned}
$$

so that we find

$$
\begin{aligned}
\hat{D}_{\delta \bar{X}_{\perp}} K_{A B}{ }^{i}= & -\widetilde{\mathcal{D}}_{A} \widetilde{\mathcal{D}}_{B} \phi^{i}+\left[K_{A C}{ }^{i} K^{C}{ }_{B j}+K_{A}{ }^{i} K_{B j} \phi^{j}\right] \\
& +K_{A C}{ }^{i} k^{C}{ }_{B} \psi+\left(\widetilde{\mathcal{D}}_{A} K_{B}{ }^{i}\right) \psi+2 K_{(A}{ }^{i} \mathcal{D}_{B)} \psi .
\end{aligned}
$$

Finally, for the deformation of its trace over the edge indices we obtain

$$
\begin{aligned}
\hat{D}_{\delta \bar{X}_{\perp}}\left(h^{A B} K_{A B}{ }^{i}\right)= & -\widetilde{\mathcal{D}}_{A} \widetilde{\mathcal{D}}^{A} \phi^{i}-\left[K_{A B}{ }^{i} K^{A B j}-K_{A}{ }^{i} K^{A}{ }_{j}\right] \phi^{j} \\
& -K_{A B}{ }^{i} k^{A B} \psi-\left(\widetilde{\mathcal{D}}_{A} K^{A i}\right) \psi+2 K_{A}{ }^{i} \mathcal{D}^{A} \psi .
\end{aligned}
$$

This is all we need for DNG extended objects with DNG edges. It is straightforward to use this method to obtain the expressions for the deformation of the remaining geometrical structures on the boundary.

Let us return now to the promised explanation of the significance of the hat on the deformation operator $D_{\bar{X}_{\perp}}$. It is related to its covariance transformation properties under normal rotations.

First, we consider the following calculational check on Eq. (30). If we let $\phi^{i}=0$ in Eq. (30), that is if we specialize to pure normal deformations of the edge extrinsic curvature (into $m$ ), we should get agreement with the normal deformation of the extrinsic curvature of $\partial m$, seen as an hypersurface in $m$. By specializing Eq.(4.6) of Ref. [9] to the case of an hypersurface, this is given by

$$
D_{\delta \chi_{\perp}} k_{A B}=-\mathcal{D}_{A} \mathcal{D}_{B} \psi+\left[k_{A C} k_{B}^{C}-\mathcal{R}_{a c b d} \epsilon_{A}^{a} \epsilon_{B}^{b} \eta^{c} \eta^{d}\right] \psi,
$$

where $\mathcal{R}_{a b c d}$ denotes the Riemann curvature of $m$. There is no twist and therefore no ambiguity in the definition of $D_{\delta \chi_{\perp}}:=\psi \eta^{\mu} D_{\mu}$.

To compare, we exploit the bulk Gauss-Codazzi equation to express the worldsheet Riemann tensor projection appearing in Eq.(36) in terms of the appropriate projections of worldsheet extrinsic curvature quadratics:

$$
\mathcal{R}_{a c b d} \epsilon_{A}^{a} \epsilon_{B}^{b} \eta^{c} \eta^{d}-K_{A B}{ }^{i} K_{c d i} \eta^{c} \eta^{d}+K_{A}{ }^{i} K_{B i}=0 .
$$

We note that the middle term vanishes for a DNG solution modulo the bulk equations of motion (11), together with the boundary conditions (31). The last term coincides with an equal term appearing in the decomposition of the Hessian (29). We now have, using this expression to eliminate the worldsheet Riemann curvature, that

$$
D_{\delta \chi_{\perp}} k_{A B}=-\mathcal{D}_{A} \mathcal{D}_{B} \psi+\left[k_{A C} k^{C}{ }_{B}+K_{A}{ }^{i} K_{B i}-K_{A B}{ }^{i} K_{c d i} \eta^{c} \eta^{d}\right] \psi .
$$

Comparing now (36) when $\phi^{i}=0$, with (38), the existence of the last term in the right hand side of Eq. (38) would appear to point to an inconsistency. It is clearly not legitimate at this level to invoke the bulk equations of motion to disregard it.

In fact, there is no inconsistency. Here is where the hat appearing on the deformation operator $D_{\delta \bar{X}_{\perp}}$ comes in. Recall that for a field transforming as a tensor under normal 
rotations, such as $L_{A B}{ }^{I}$, the construction of a totally covariant measure of the deformation $\hat{D}_{\bar{X}_{\perp}}$ involves the addition of a deformation connection [9]:

$$
\hat{D}_{\delta \bar{X}_{\perp}} L_{A B}^{I}=D_{\delta \bar{X}_{\perp}} L_{A B}^{I}-\hat{\gamma}^{I J} L_{A B J}
$$

The connection is given by

$$
\hat{\gamma}^{I J}=g\left(D_{\delta \bar{X}_{\perp}} m^{I}, m^{J}\right)
$$

This connection is necessary to ensure manifest covariance under the full $O(N-D+1)$ normal rotations. However, as emphasized above, we are breaking the invariance down to $O(N-D)$, when we surrender the freedom to rotate the $\eta^{\mu}$ into the $n^{\mu i}$. This fact is responsible for the appearance of the extra term in Eq. (38).

Let us, now, verify explicitly that the additional term appearing in Eq.(38) indeed coincides with the deformed connection correction. We have

$$
\hat{D}_{\bar{X}_{\perp}} k_{A B}=D_{\bar{X}_{\perp}} k_{A B}-\hat{\gamma}^{0 i} K_{A B i} .
$$

Using the "deformation Gauss-Weingarten equations" [9], a short calculation gives

$$
\hat{\gamma}^{0 i}=-K_{a b i} \eta^{a} \eta^{b} \psi+\eta \cdot \tilde{\nabla} \phi_{i}
$$

The first term coincides with the offending term appearing in Eq.(38). The latter term involving normal gradients of the $\phi^{i}$ is of a form which does not appear elsewhere in Eq.(30), but that vanishes anyway when we consider pure normal deformations of the edge into $m$.

It was argued incorrectly in Ref. [9] that the deformation connection could be gauged away in general. We are presenting here an explicit counterexample to that claim. The argument outlining the circumstances under which it could be ignored was, however, correct. We always envisaged the application of the formalism to quantities which vanish on shell, such as, for example, $K^{i}$. Also here, on shell, the deformation connection does not contribute for example to the deformation of the mean extrinsic curvature of the edges, $k$, that is $\hat{D}_{\delta \bar{X}_{\perp}} k=D_{\delta \bar{X}_{\perp}} k$.

Similarly, for the deformation of the remaining projections, $L_{A B}{ }^{i}$, one has that

$$
\hat{D}_{\delta \bar{X}_{\perp}} K_{A B}^{i}=D_{\delta \bar{X}_{\perp}} K_{A B}^{i}-\hat{\gamma}^{i j} K_{A B j}-\hat{\gamma}^{i 0} k_{A B}
$$

Noting that

$$
\hat{\gamma}^{i j}=\gamma^{i j}+\psi \eta^{a} \omega_{a}^{i j}
$$

and using Eq. (42), one obtains

$$
\left.\hat{D}_{\delta \bar{X}_{\perp}} K_{A B}^{i}=\widetilde{D}_{\delta \bar{X}_{\perp}} K_{A B}^{i}+\left[(\eta \cdot \tilde{\nabla}) \phi^{i}+K_{a b}^{i} \eta^{a} \eta^{b}\right] k_{A B}+\psi \omega_{a}^{i j} \eta^{a}\right) K_{A B j}
$$

For the deformation of its trace over the edge indices, we obtain then

$$
\hat{D}_{\delta \bar{X}_{\perp}}\left(h^{A B} K_{A B}^{i}\right)=\widetilde{D}_{\delta \bar{X}_{\perp}}\left(h^{A B} K_{A B}^{i}\right)+\left[(\eta \cdot \tilde{\nabla}) \phi^{i}+K_{a b}^{i} \eta^{a} \eta^{b}\right] k
$$




\section{LINEARIZED EQUATIONS OF MOTION}

It is straightforward at this point to read off the linearized equations of motion. We have the linearized equation in the bulk, or $\widetilde{D}_{\delta X_{\perp}} K^{i}=0$, given by [6]

$$
\tilde{\Delta} \Phi^{i}+K_{a b}^{i} K^{a b}{ }_{j}^{j} \Phi^{j}=0
$$

The linearizations of Eq.(2) and Eq.(3) are respectively, $\hat{D}_{\delta \bar{X}_{\perp}} k=0$, and $\hat{D}_{\delta \bar{X}_{\perp}} h^{A B} K_{A B}^{i}=$ 0 , or

$$
\begin{aligned}
\mathcal{D}^{A}\left(\mathcal{D}_{A} \psi+K_{A}^{i} \phi_{i}\right)+K_{i}^{A}\left(\widetilde{\mathcal{D}}_{A} \phi^{i}-K_{A}^{i} \psi\right)+k^{A B}\left(k_{A B} \psi+K_{A B}^{i} \phi_{i}\right) & =0, \\
\widetilde{\mathcal{D}}^{A}\left(\widetilde{\mathcal{D}}_{A} \phi^{i}-K_{A}^{i} \psi\right)-K^{A i}\left(\mathcal{D}_{A} \psi+K_{A}^{j} \phi_{j}\right)+K_{A B}^{i}\left(K^{A B j} \phi_{j}+k^{A B} \psi\right) & =0 .
\end{aligned}
$$

In this form, a symmetry in the boundary conditions becomes manifest which is not apparent in their original form.

If the parent worldsheet is totally geodesic, so that $K_{a b}^{i}=0$, then these equations reduce to

$$
\begin{gathered}
\tilde{\Delta} \Phi^{i}=0 \\
\left(\mathcal{D}^{A} \mathcal{D}_{A}+k^{A B} k_{A B}\right) \psi=0, \\
\widetilde{\mathcal{D}}^{A} \widetilde{\mathcal{D}}_{A} \phi^{i}=0 .
\end{gathered}
$$

Then $\phi^{i}$ and $\psi$ completely decouple. An example is provided by a non - rotating straight length of string bounded by particles, or a flat disc of membrane bounded by a string. In the former case, we have $\ddot{\psi}-(\mu / M)^{2} \psi=0$ and $\ddot{\phi}^{i}=0$ on the end, where dot represents the derivative with respect to proper time along the trabectory of the end particle of mass $M$ (also see next section). The tension in the bulk accelerates the end particles uniformly, it is necessarily destabilizing. We will discuss this issue in the next section.

Does any analogous behavior prevail when $K_{a b}^{i} \neq 0$ ? Suppose we have $\Phi^{i}=0=\phi^{i}$ everywhere. Then the linearized equations of motion reduce to Eq. (51), and

$$
\begin{aligned}
{\left[\mathcal{D}^{A} \mathcal{D}_{A}+k^{A B} k_{A B}-K^{A i} K_{A i}\right] \psi } & =0, \\
2 K^{A i} \mathcal{D}_{A} \psi-\left(\widetilde{\mathcal{D}}_{A} K_{A}^{i}\right) \psi+K_{A B}^{i} k^{A B} \psi & =0 .
\end{aligned}
$$

In general, the only solution of Eq.(54) which is consistent with the Eq.(53) is the trivial solution, $\psi=0$. Pure edge states do not generally exist. Conversely, pure bulk states with $\psi=0$ are also impossible.

Of course, there remains the possibility to fix the ends with $\psi=\phi^{i}=0$, and to consider only bulk deformations, given by solutions of (47) with Dirichlet-type boundary conditions at the ends. 


\section{STRING BOUNDED BY POINT PARTICLES}

In this section, we specialize the general treament given above, to the case of a string with massive point particles attached to its ends.

We parametrize the boundary worldline by proper time, $\tau$. We denote the corresponding unit velocity vector with $v^{a}$. On the boundary, $\partial m$, the string worldsheet metric can be parametrized as

$$
\gamma^{a b}=-v^{a} v^{b}+\eta^{a} \eta^{b}
$$

where recall that $\eta^{a}$ is the normal to $\partial m$ into $m$.

We define

$$
\begin{aligned}
K_{\|\|}^{i} & :=K_{a b}^{i} v^{a} v^{b}, \\
K_{\perp \|}^{i} & :=K_{a b}^{i} \eta^{a} v^{b}, \\
K_{\perp \perp}^{i} & :=K_{a b}^{i} \eta^{a} \eta^{b} .
\end{aligned}
$$

The string background equations of motion, evaluated at the boundary, then imply

$$
K_{\perp \perp}^{i}=K_{\|\|}^{i}
$$

whereas the boundary conditions, Eq.(3), imply that

$$
K_{\|\|}^{i}=0 \text {, }
$$

and as a result also

$$
K_{\perp \perp}^{i}=0 .
$$

Thus, we can now express the extrinsic curvature of the worldsheet $m$ on the boundary in the off-diagonal form,

$$
K_{a b}^{i}=-K_{\perp \|}^{i}\left(\eta_{a} v_{b}+\eta_{b} v_{a}\right) .
$$

We then have on the boundary

$$
K_{a b}{ }^{i} K^{a b}{ }_{j}=-2 K_{\perp \|}{ }^{i} K_{\perp \| j} \leq 0 .
$$

The latter implies that the mass matrix appearing in the linearized bulk equations of equation, Eq. (47), has no negative eigenvalues, corresponding to tachyonic modes, in the neighborhood of the boundary.

We also note that the extrinsic curvature associated with the embedding of the end worldline in the string worldsheet is identified with the geodesic curvature which is completely determined by the boundary conditions. We do not need to solve Eq.(2) explicitly. The boundary conditions, Eqs.(48) and (49), reduce to the system of coupled ordinary differential equations:

$$
\begin{gathered}
\ddot{\psi}-\left[K_{\perp \|}^{i} K_{\perp \| i}+k^{2}\right] \psi+2 K_{\perp \|}{ }^{i} \dot{\phi}_{i}+\dot{K}_{\perp \|}{ }^{i} \phi_{i}-\omega_{\| i j} K_{\perp \|}{ }^{i} \phi^{j}=0, \\
\ddot{\phi}^{i}-K_{\perp \|}{ }^{i} K_{\perp \|}{ }^{j} \phi_{j}-2 K_{\perp \|}{ }^{i} \dot{\psi}-\dot{K}_{\perp \|}{ }^{i} \psi+\omega_{\| i j} K_{\perp \|}{ }^{i} \phi^{j}=0,
\end{gathered}
$$

where $\omega_{\| i j}:=\eta^{a} \omega_{a i j}$. The mass terms appearing in the boundary conditions should be contrasted with those in the bulk equations. Their diagonal entries are explicitly tachyonic. This does not, in itself, however, signal the non-existence of harmonic perturbations. One has to take into account both the off diagonal terms (coupling $\psi$ and $\phi^{i}$ ) and the first derivative terms. 


\section{RIGIDLY ROTATING STRING BOUNDED BY POINT PARTICLES}

Consider a string bounded by two pointlike masses rotating rigidly in a plane [4]. We describe Minkowski space by cylindrical polar coordinates, $(t, r, \theta, z)$. The worldsheet generated by the motion of the string can be described by the embedding in Minkowski space,

$$
X^{\mu}(t, r)=(t, r \cos \theta(t), r \sin \theta(t), 0) .
$$

This embedding induces a line element on the string worldsheet given by

$$
d s^{2}=-\left(1-r^{2} \dot{\theta}^{2}\right) d t^{2}+d r^{2}
$$

for $0 \leq r \leq R(t)$

We now evaluate the extrinsic curvature tensor which corresponds to the worldsheet described by Eq.(67). It is clear that the only non-vanishing normal component is that which corresponds to the normal which lies in the plane of motion. We have

$$
\begin{aligned}
K_{t t} & =\frac{-r \ddot{\theta}}{\left(1-r^{2} \dot{\theta}^{2}\right)^{1 / 2}} \\
K_{r t} & =\frac{-\dot{\theta}}{\left(1-r^{2} \dot{\theta}^{2}\right)^{1 / 2}} \\
K_{r r} & =0 .
\end{aligned}
$$

The extrinsic twist for this embedding is identically zero, $\omega_{a}^{i j}=0$.

The string equation of motion reduces to $K_{t t}=0$ or $\ddot{\theta}=0$ with solution $\theta=\omega_{0} t+\theta_{0}$. This corresponds to a right circular timelike helicoid, which is null when $\omega_{0} r=1$.

The proper time along the boundary of the worldsheet at $r=R(t)$ is in turn given by

$$
d \tau^{2}=\left(1-\dot{R}^{2}-R^{2} \omega_{0}^{2}\right) d t^{2} .
$$

The tangent vector to the boundary at $r=R(t)$ is given by

$$
v^{a}(\tau)=\frac{1}{\sqrt{1-\omega_{0}^{2} R^{2}+\dot{R}^{2}}}(1, \dot{R}),
$$

while the normal into $m$ of the boundary is

$$
\eta^{a}(\tau)=\frac{1}{\sqrt{1-\omega_{0}^{2} R^{2}} \sqrt{1-\omega_{0}^{2} R^{2}+\dot{R}^{2}}}\left(\dot{R}, 1-R^{2} \omega_{0}^{2}\right)
$$

The projections of the parent worldsheet extrinsic curvature onto the boundary is

$$
K_{\|\|}=-\frac{1}{\left(1-\dot{R}^{2}-R^{2} \omega_{0}^{2}\right) \sqrt{1-R^{2} \omega_{0}^{2}}} 2 \omega_{0} \dot{R},
$$

so that the boundary condition implies that $R$ is constant (see Eq. (60)).

We conclude that the equations of motion place a very stringent restriction on the possible motion. In particular, Newtonian intuition is misleading. It would suggest that rigid motion 
is possible with variable $\dot{\theta}$ and variable $R$. After all the particles at the ends themselves are subject to a linear potential, the rotation supplying a centrifugal repulsion so that we have a straighforward Kepler problem. Indeed, one can formulate a Nambu theory for this system with a richer configuration space. It is obtained by introducing (67) and (69) directly at the level of the action [10].

Modulo the boundary conditions, one finds that $K_{\perp \perp}=0$, as was to be expected, and that the mixed projection is given by

$$
K_{\perp \|}=\frac{-\omega_{0}}{\left(1-R^{2} \omega_{0}^{2}\right)^{2}} .
$$

Finally, using that $R$ and $\dot{\theta}$ are constants, the geodesic curvature of the ends is,

$$
k=-\frac{R \omega_{0}^{2}}{1-R^{2} \omega_{0}^{2}}
$$

so that the ends equation of motion is

$$
M R \omega_{0}^{2}=\mu\left(1-R^{2} \omega_{0}^{2}\right) .
$$

Let us now examine arbitrary small perturbations about such timelike circular helicoids. We have that

$$
K_{a b}^{i} K_{j}^{a b}=-2 \delta_{1}^{i} \delta_{j}^{1} \frac{\omega_{0}^{2}}{\left(1-\omega_{0}^{2} r^{2}\right)^{2}} .
$$

The mass matrix appearing in (47) has one positive eigenvalue. Note that in the limit, $r \rightarrow R$, and $\omega_{0} R \rightarrow 1$, this curvature invariant diverges. The worldsheet is singular in the neighborhood of its null boundary. It is not surprising: there are no curvature penalties in the DNG dynamics. The DNG approximation breaks down.

We can now exploit a conformal coordinate system on the helicoid to simplify the form of the d'Alembertian appearing in (47). We have

$$
\begin{aligned}
d s^{2} & =\left(1-\omega_{0}^{2} r^{2}\right)\left(-d t^{2}+\frac{d r^{2}}{1-\omega_{0}^{2} r^{2}}\right) \\
& =\cos ^{2}\left(\omega_{0} X\right)\left(-d t^{2}+d X^{2}\right),
\end{aligned}
$$

where

$$
\omega_{0} X=\arcsin \left(\omega_{0} r\right) .
$$

Thus, the bulk linearized equations of motion reduce to

$$
\begin{aligned}
\frac{\partial^{2} \Phi_{1}}{\partial t^{2}}-\frac{\partial^{2} \Phi_{1}}{\partial X^{2}}+\frac{2 \omega_{0}^{2}}{\cos ^{2}\left(\omega_{0} X\right)} \Phi_{1} & =0, \\
\frac{\partial^{2} \Phi_{2}}{\partial t^{2}}-\frac{\partial^{2} \Phi_{2}}{\partial X^{2}} & =0,
\end{aligned}
$$

Note that they are completely decoupled, and that the solutions are well behaved. On a solution without dynamical boundary, the solution appears to be stable. However, the 
geometry is singular at the null edge and it is not obvious that perturbation theory makes sense.

On the boundary, we have, with $\phi:=\phi_{1}$,

$$
\begin{gathered}
\ddot{\psi}-\left(K_{\perp \|}^{2}+k^{2}\right) \psi+2 K_{\perp \|} \dot{\phi}=0, \\
\ddot{\phi}-K_{\perp \|}^{2} \phi-2 K_{\perp \|} \dot{\psi}=0, \\
\ddot{\phi}_{2}=0 .
\end{gathered}
$$

Let

$$
\begin{aligned}
& \psi=\psi_{0} e^{-i \omega \tau}, \\
& \phi=\phi_{0} e^{-i \omega \tau} .
\end{aligned}
$$

We then have

$$
\begin{aligned}
\left(\omega^{2}+K_{\perp \|}^{2}+k^{2}\right) \psi_{0}+2 i \omega K_{\perp \|} \phi_{0} & =0 \\
\left(\omega^{2}+K_{\perp \|}^{2}\right) \phi_{0}-2 i \omega K_{\perp \|} \psi_{0} & =0 .
\end{aligned}
$$

A non-trivial solution exists when

$$
\omega^{4}+\left[k^{2}-2 K_{\perp \|}^{2}\right] \omega^{2}+\left[k^{2}+K_{\perp \|}^{2}\right] K_{\perp \|}^{2}=0 .
$$

When we substitute the values (73) and (74), we find that all four eigenfrequencies are complex. This implies that no analogue of the breathing modes exists in the DNG spectrum.

The linearized theory is unusual in two related respects. There is no feedback on $\partial m$ from perturbations in the bulk. The boundary equations of motion can be solved without reference to the bulk motion. In particular, the two boundaries behave independently at this order. The physical reason for this is the weak nature of the coupling between the string and the end particles. The force which binds them is constant, not harmonic.

\section{CONCLUSIONS}

In this paper, we have derived the complete linearized equations of motion for a relativistic DNG membrane with DNG edges. One direct physical application of these equations is to examine the stability of the QCD string with massive quarks at its ends. For rigidly rotating configurations, we calculate the normal modes and we find that, in general, they are complex. Whether this implies a genuine instability in the system remains to be demonstrated. The necessary classical ingredient in the determination of the effect of quantum fluctuations in a saddle point evaluation of the path integral is the calculation of the second order variation of the action. This variation can be read off straightforwardly from the expressions derived in this paper.

Finally, we mention that relativistic extended objects with edges provide a simplified well defined setting in which to examine the relationship between bulk and boundary degrees of freedom that is thought to be relevant in various contexts in gravitational physics, most notably that of black holes. 


\section{ACKNOWLEDGMENTS}

We have benefitted from conversations with Brandon Carter, Xavier Martin and Alexander Vilenkin. We gratefully acknowledge support from CONACyT grant no. 211085-50118PE. JG would like to thank Prof. J. Lewis of the Dublin Institute for Advanced Studies for hospitality during his stay in Dublin.

\section{APPENDIX}

In this appendix, we give a brief list of the equations corresponding to the case of an arbitrary background spacetime. We denote with $R_{\nu \rho \sigma}^{\mu}$ the Riemann curvature tensor of the background spacetime.

The extrinsic curvature of the edge as embedded in spacetime transforms now as

$$
\hat{D}_{\delta \bar{X}_{\perp}} L_{A B}{ }^{I}=-\hat{\mathcal{D}}_{A} \hat{\mathcal{D}}_{B} \phi^{I}+L_{A C}{ }^{I} L^{C}{ }_{B J} \phi^{J}-R_{\mu \nu \sigma \rho} f^{\mu}{ }_{A} f^{\sigma}{ }_{B} m^{\nu I} m^{\rho}{ }_{J} \phi^{J} .
$$

The additional term involving the background curvature propagates in the equations corresponding to the projections as

$$
\begin{aligned}
\hat{D}_{\delta \bar{X}_{\perp}} k_{A B}= & -\mathcal{D}_{A} \mathcal{D}_{B} \psi+\left[K_{A}^{i} K_{i B}+k_{A C} k_{B}^{C}-R_{\mu \nu \sigma \rho} f^{\mu}{ }_{A} f_{B}^{\sigma} \eta^{\nu} \eta^{\rho}\right] \psi \\
& -2 K^{i}{ }_{(A} \widetilde{\mathcal{D}}_{B)} \phi_{i}-\left(\widetilde{\mathcal{D}}_{A} K_{B}^{i}\right) \phi_{i}+k_{A C} K_{B i}^{C} \phi^{i}-R_{\mu \nu \sigma \rho} f^{\mu}{ }_{A} f^{\sigma}{ }_{B} \eta^{\nu} n^{\rho}{ }_{i} \phi^{i}
\end{aligned}
$$

and

$$
\begin{aligned}
\hat{D}_{\delta \bar{X}_{\perp} K_{A B}{ }^{i}=} & -\widetilde{\mathcal{D}}_{A} \widetilde{\mathcal{D}}_{B} \phi^{i}+\left[K_{A C}{ }^{i} K^{C}{ }_{B j}+K_{A}{ }^{i} K_{B j}-R_{\mu \nu \sigma \rho} f^{\mu}{ }_{A} f^{\sigma}{ }_{B} n^{\nu 1} n^{\rho}{ }_{j}\right] \phi^{j} \\
& +K_{A C}{ }^{i} k^{C}{ }_{B} \psi+\left(\widetilde{\mathcal{D}}_{A} K_{B}{ }^{i}\right) \psi+2 K_{(A}{ }^{i} \mathcal{D}_{B)} \psi-R_{\mu \nu \sigma \rho} f^{\mu}{ }_{A} f^{\sigma}{ }_{B} n^{\nu i} \eta^{\rho} \psi .
\end{aligned}
$$

For the traces over the edge indices, we obtain:

$$
\begin{aligned}
\hat{D}_{\delta \bar{X}_{\perp} k==} & -\mathcal{D}_{A} \mathcal{D}^{A} \psi+\left[K_{A i} K^{A i}-k_{A B} k^{A B} R_{\mu \nu \sigma \rho} f^{\mu}{ }_{A} f^{\sigma A} \eta^{\nu} \eta^{\rho}\right] \psi \\
& -2 K_{A}{ }^{i}\left(\widetilde{\mathcal{D}}^{A} \phi_{i}\right)-\left(\widetilde{\mathcal{D}}_{A} K^{A i}\right) \phi_{i}-k_{A B} K^{A B}{ }_{i} \phi^{i} \\
& -R_{\mu \nu \sigma \rho} f^{\mu}{ }_{A} f^{\sigma B} \eta^{\nu} n^{\rho}{ }_{i} \phi^{i}
\end{aligned}
$$

and

$$
\begin{aligned}
\hat{D}_{\delta \bar{X}_{\perp}}\left(h^{A B} K_{A B}{ }^{i}\right)= & -\widetilde{\mathcal{D}}_{A} \widetilde{\mathcal{D}}^{A} \phi^{i}-\left[K_{A B}{ }^{i} K^{A B j}-K_{A}{ }^{i} K^{A}{ }_{j}-R_{\mu \nu \sigma \rho} f^{\mu}{ }_{A} f^{\sigma B} n^{\nu 1} n^{\rho}{ }_{j}\right] \phi^{j} \\
& -K_{A B}{ }^{i} k^{A B} \psi-\left(\widetilde{\mathcal{D}}_{A} K^{A i}\right) \psi+2 K_{A}{ }^{i} \mathcal{D}^{A} \psi \\
& -R_{\mu \nu \sigma \rho} f^{\mu}{ }_{A} f^{\sigma B} n^{\nu i} \eta^{\rho} \psi
\end{aligned}
$$

The linearized equations of motion for the bulk are given by [6]:

$$
\tilde{\Delta} \Phi^{i}+\left[K_{a b}{ }^{i} K_{j}^{a b}-R_{\mu \nu \sigma \rho} e^{\mu}{ }_{a} e^{\sigma a} n^{\nu i} n^{\rho}{ }_{j}\right] \Phi^{j}=0,
$$

and, finally, the linearized equations of motion for the edges are obtained by simply setting to zero the right hand sides of Eqs. (89), (90). 


\section{REFERENCES}

[1] R. Capovilla and J. Guven, Rev. Mex. de Fisica 41765 (1995)

[2] R. Capovilla and J. Guven, Phys. Rev. D55 2388 (1997)

[3] B. Carter, in Formation and Interactions of Topological Defects (NATO ASI B349), ed R. Brandenberger \& A.C. Davis, pp 303-348 (Plenum, New York, 1995).

[4] B.M. Barbashov and V.V. Nesterenko Introduction to Relativistic String Theory (World Scientific, Singapore, 1989)

[5] A. Vilenkin and E.P.S. Shellard Cosmic Strings and Other Topological Defects (Cambridge Univ. Press, Cambridge, 1994)

[6] J. Guven, Phys Rev D48 5562 (1993);

[7] A.L. Larsen and V.P. Frolov, Nucl Phys B414, 129 (1994).

[8] B. Carter, Phys. Rev. D48, 4835 (1993).

[9] R. Capovilla and J. Guven, Phys. Rev. D51 6745 (1995)

[10] G. Arreaga, R. Capovilla, and J. Guven, in preparation. 